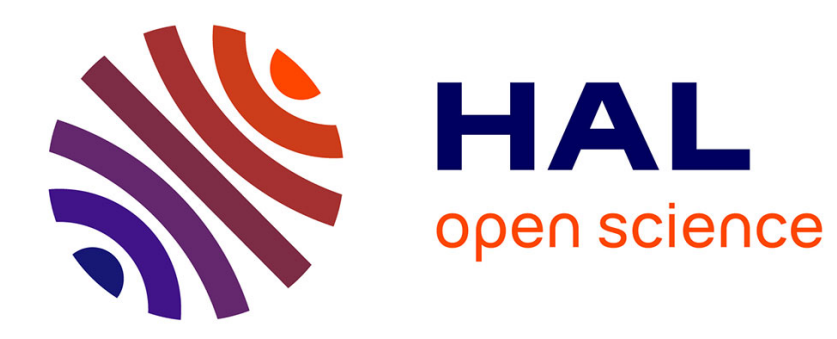

\title{
Recherches expérimentales sur la diffraction
}

\author{
M. Gouy
}

\section{To cite this version:}

M. Gouy. Recherches expérimentales sur la diffraction. J. Phys. Theor. Appl., 1887, 6 (1), pp.32-38.

10.1051/jphystap:01887006003200 . jpa-00238739

\section{HAL Id: jpa-00238739 https://hal.science/jpa-00238739}

Submitted on 1 Jan 1887

HAL is a multi-disciplinary open access archive for the deposit and dissemination of scientific research documents, whether they are published or not. The documents may come from teaching and research institutions in France or abroad, or from public or private research centers.
L'archive ouverte pluridisciplinaire HAL, est destinée au dépôt et à la diffusion de documents scientifiques de niveau recherche, publiés ou non, émanant des établissements d'enseignement et de recherche français ou étrangers, des laboratoires publics ou privés. 


\section{REGHERGHES EXPÉRIMENTALES SUR LA DIFFRAGTION;}

Par M. GOUY ( $\left.{ }^{1}\right)$.

\section{$\S$ I. - Objet du Mémoire et méthode expérimentale.}

La diffraction de la lumière n'avait été étudiée jusqu'ici que dans le cas limite où les rayons diffractés sont déviés d'un très petit angle et ne s'éloignent que très peu de la direction des rayons incidents $\left({ }^{2}\right)$. Si l'on écarte cette restriction, en examinant des rayons déviés d'un grand angle par la diffraction, on se trouve en présence de phénomènes nouveaux, qui font l'objet du travail que nous allons résumer.

Méthode expérimentale. - On projette l'image d'une source Fig. I.

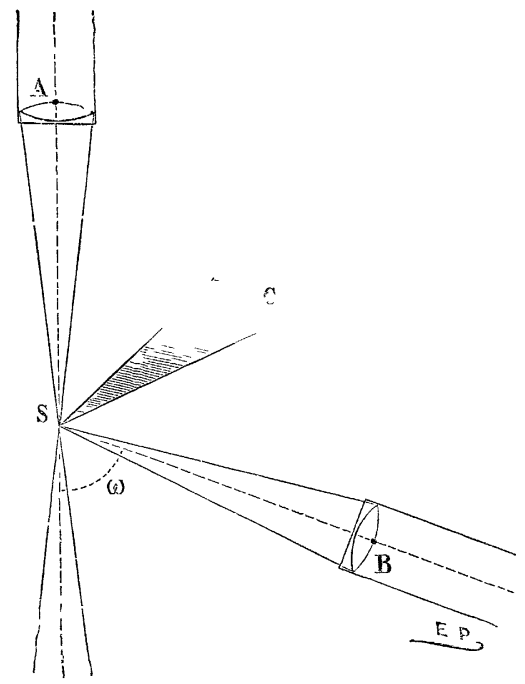

lumineuse intense (Soleil, arc électrique), sur le bord d'un écran

( $\left.{ }^{2}\right)$ Le Mémoire complet a paru dans les Annales de Chimie et de Physique, $6^{\text {e }}$ série, t. VIII, juin r 886 .

$\left({ }^{2}\right)$ Sauf pourtant avec les réseaux qui donnent, comme on sait, des résultats variables et très complexes, au point de vue de la polarisation des rayons diffractés. 
opaque (1). On observe les rayons diffractés au moyen d'un microscope à long foyer mis au point sur ce bord lui-même. On voit dans le champ du microscope une ligne lumincuse très brillante, qui dessine le bord de l'écran, et qui est formée par la lumière diffractée. La $f i g$. I montre l'appareil en projection horizontale, le bord de l'écran étant vertical ( $\mathrm{A}$, lentille de projection, achromatique; $\mathrm{S}$, image de la source; $\mathrm{SC}$, écran; $\mathrm{B}$, objectif du microscope; $\omega$, déviation des rayons reçus dans l'instrument).

\section{§II. - Diffraction intérieure à l'ombre géométrique.}

Dans la position de la $f i g$. ז, les rayons diffractés, pour arriver à l'observateur, contournent le bord de l'écran et pénètrent dans l'ombre géométrique, sans mélange possible de lumière réfléchie. Leur intensité diminue quand la déviation $\omega$ augmente, d'abord rapidement, puis de moins en moins vite; on peut encore observer aisément, avec presque tous les métaux, des rayons déviés de $150^{\circ}$ à $\mathbf{} 60^{\circ}$, et possédant, malgré cette grande déviation, une intensité assez notable. Aucune particularité ne distingue la direction où les rayons diffractés sont perpendiculaires aux rayons incidents. L'intensité est indépendante de la nature de l'écran, lorsque la déviation est très petite; mais, quand celle-ci est notable, l'intensité est d'autant moindre que le bord est plus arrondi. Elle dépend aussi de la nature du métal; ainsi l'argent donne environ quatre fois plus de lumière que l'acier, pour une déviation de $45^{\circ}$.

Colorations des rayons diffractés. - La lumière incidente étant blanche, les rayons diffractés présentent des colorations souvent très vives, quand la déviation n'est pas très petite, et variables suivant le métal qui forme l'écran. Avec l'acier et la plupart des corps peu colorés et d'un médiocre pouvoir réflecteur, la lumière diffractée est blanche, ou un peu jaunâtre quand

( ${ }^{1}$ Les écrans, en général métalliques, doivent avoir un bord bien régulier, ou tranchant, ou légèrement arrondi, ce qui modifie les phénomènes. L'épaisseur du bord doit toujours être très petite; une épaisseur de $\frac{\mathrm{I}^{\mathrm{mm}}}{\mathrm{IOO}}$ est déjà trop considérable pour de très grandes déviations.

J. de Phys., $2^{\circ}$ série, t. VI. (Janvier 1887 .) 
la déviation est très grande et le bord arrondi. L'argent donne de la lumière blanche ou peu colorée avec un bord tranchant; avec un bord arrondi, on a des alternances de teintes assez vives, variables avec la déviation, allant du jaune vert au rouge; ces teintes sont d'autant plus vives qu'elles sont moins réfrangibles. Quand le bord est plus arrondi, elles passent à l'orangé et au rouge et prennent une grande intensité. Plusieurs métaux sensiblement blancs se comportent à peu près comme l'argent. Les métaux rouges (cuivre, or) ne donnent que des teintes orangé ou rouge, qui deviennent très pures quand le bord est un peu arrondi; ces teintes rappellent la couleur superficielle du métal, exagérée comme elle le serait après plusieurs réflexions; analysées au spectroscope, elles paraissent presque monochromatiques. La manière dont se comporte chaque métal est caractéristique, presque indépendante de la manière dont le bord a été travaillé, quoique les bords bien réguliers donnent seuls des teintes très pures.

Polarisation des rayons diffractés. - La lumière incidente étant naturelle, les rayons diffractés à l'intérieur de l'ombre possèdent une polarisation rectiligne partielle, parallèlement au bord de l'écran, c'est-à-dire perpendiculairement au plan de diffraction, qui contient les rayons incidents et les rayons diffractés. Cette polarisation, insensible pour de petites déviations, s'accroît très rapidement quand la déviation augmente, devient bientôt presque complète, et se maintient ainsi jusqu'à la limite des expériences. Avec tous les métaux qui peuvent donner un bord bien régulier, on obtient une polarisation complète à quelques dixmillièmes près, dès que la déviation atteint $80^{\circ}$ ou $90^{\circ}$.

Il importait de rechercher si cette action polarisatrice de la diffraction intérieure, d'une énergie si remarquable, ne dépendait pas de quelque particularité due au travail du bord de l'écran; pour cela on a examiné des écrans dont les arêtes étaient formées par des moyens très divers, soit par aiguisage, soit, pour les métaux mous, au moyen d'une lame tranchante, soit par rupture ou clivage. On a aussi utilisé les arêtes naturelles de cristaux opaques. Le phénomène a toujours été de même sens, très marqué, et les différences d'intensité de la polarisation paraissent dues principalement au plus ou moins de régularité de l'arète. Le noir de fumée 
fait seul exception et ne donne qu'une polarisation insensible, lorsqu'il est bien mat.

Ces expériences présentent une particularité très digne d'atten. tion. Lorsqu'on étudie la lumière diffractée au moyen d'un analyseur biréfringent, dont la section principale est perpendiculaire au bord de l'écran, on constate que les deux images présentent des teintes fort différentes. La plus intense, polarisée parallèlement au bord de l'écran, présente les mêmes teintes vives que la lumière diffractée, prise dans son ensemble; l'autre image est toujours parfaitement blanche, avec tous les métaux, pourvu que le bord soit bien régulier. Avec le cuivre, par exemple, l'image intense est d'un rouge pur, et l'image faible est blanche, ou paraît un peu verdâtre par un effet de contraste physiologique. Ainsi la cause, quelle qu'elle soit, qui colore si vivement la lumière diffractée, n'agit pas sur sa composante perpendiculaire au bord de l'écran.

Lumière incidente polarisée. - Lorsque la lumière incidente est polarisée parallèlement ou perpendiculairement au bord de l'écran, il en est de même de la lumière diffractée; dans le premier cas, cette lumière est vive et présente les colorations variées que nous avons décrites précédemment; dans le second cas, elle est parfaitement blanche, et d'une intensité très faible quand la déviation est un peu grande.

Pour les autres azimuts de polarisation des rayons incidents, la diffraction produit une rotation du plan de polarisation, qui a pour effet de le rendre presque parallèle au bord de l'écran. Mais de plus, quand le bord n'est pas très tranchant, la diffraction donne de la lumière polarisée elliptiquement, en produisant une différence de marche entre les deux composantes de la lumière incidente polarisées parallèlement et perpendiculairement au bord de l'écran; la seconde de ces composantes prend l'avance. Cette différence de marche est croissante avec la déviation; elle approche beaucoup de $\frac{\lambda}{4}$ el même dépasse un peu cette valeur avec certains métaux. Il y a donc une polarisation elliptique par diffraction, qui peut devenir sensiblement circulaire. 
Milieux autres que l'aur. - Dans des liquides plus ou moins réfringents, les phénomènes généraux sont les mêmes que dans l'air. Mais, pour un écran déterminé, les effets varient; l'immersion dans un milieu réfringent les modifie comme si, dans l'air, on rendait le bord plus arrondi. Ainsi un écran d'argent à bord très fin donne, dans l'air, de la lumière presque blanche; dans l'eau, on aura la teinte jaune orangé; dans le chloroforme, plus réfringent, on aura un orangé rouge très vif; enfin, dans le sulfure de carbone, encore plus réfringent, la lumière sera d'un rouge presque pur.

§ III. - Diffraction extérieure à l'ombre géométrique.

En disposant l'expérience comme l'indique la $f g .2$, on peut

Fig. 2.

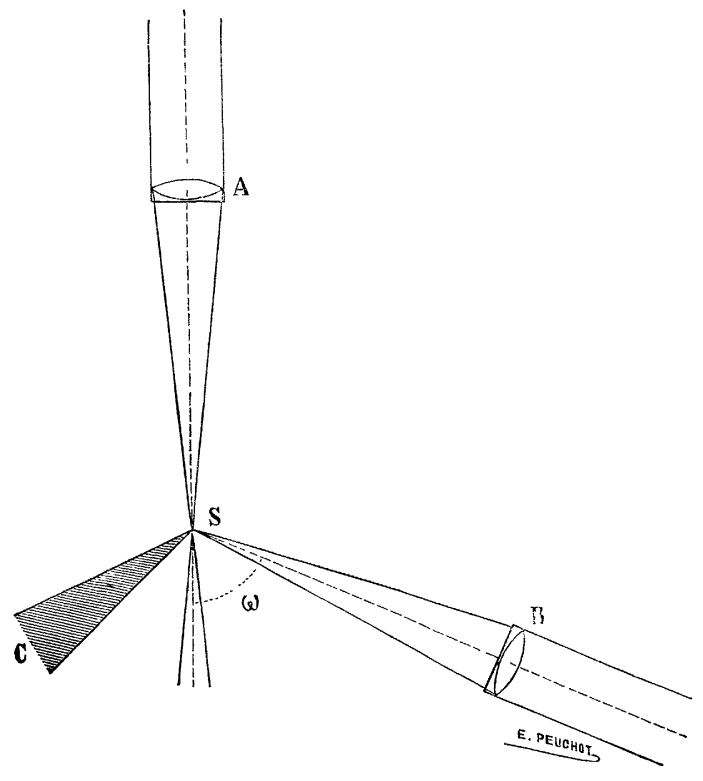

étudier la lumière diffractée à l'extérieur de l'ombre géométrique. On voit dans le champ du microscope une ligne lumineuse formée à la fois par la lumière diffractée et par celle qui est réfléchie sur 
le bord de l'écran. Des bords très tranchants donnent seuls des phénomènes notablement différents de ceux de la réflexion ordinaire et montrent les effets de la diffraction.

Avec une arête très tranchante et très régulière, l'intensité lumineuse est très grande pour une très petite déviation et décroît quand la déviation augmente, d'abord très rapidement, puis de moins en moins vite jusqu'à la limite des expériences. Pour une même déviation, l'intensité lumineuse est la même que pour la diffraction intérieure, avec la lumière incidente naturelle, comme le montre la comparaison directe.

Polarisation. - La lumière incidente étant naturelle, la lumière diffractée est polarisée perpendiculairement au bord de l'écran, c'est-à-dire parallèlement au plan de diffraction. Cette polarisation augmente avec la déviation jusque vers $30^{\circ}$ ou $40^{\circ}$, puis décroît un peu. A son maximum, avec des arêtes très tranchantes, elle est presque complète, à deux ou trois centièmes près, et quelquefois moins. Cette polarisation est de même sens que celle que donnerait la simple réflexion sur le bord de l'écran, mais hors de toute proportion avec celle-ci, toujours assez faible; on doit donc l'attribuer à la diffraction extérieure. Ainsi les deux genres de diffraction, intérieure et extérieure, donnent des polarisations très marquées, mais dans deux sens rectangulaires. Ces effets complémentaires des deux genres de diffraction, tout à fait imprévus, expliquent jusqu'à un certain point pourquoi les réseaux, où ces deux genres de diffraction existent simultanément, donnent une polarisation peu marquée et de sens variable.

Remarque. - Remarquons, en terminant, que les théories de la diffraction, données par Stokes et par d'autres auteurs, ne faisaient prévoir, ni les effets complémentaires des deux genres de diffraction, ni les colorations dépendant de la nature de l'écran, ni la polarisation elliptique produite par la diffraction intérieure. Il semble donc qu'un élément essentiel du problème avait été négligé ; en effet, les phénomènes dépendant de la nature de l'écran montrent que celui-ci ne se borne pas à arrêter une partie de l'onde incidente, et que le métal entre en relation avec le mouvement vibratoire qui se propage au voisinage immédiat de l'arête. La théorie 
de la diffraction doit tenir compte de cette relation et se trouve par là rapprochée de la théorie de la réflexion métallique; les deux genres de phénomènes présentent d'ailleurs certaines analogies, pour lesquelles nous renverrons au Mémoire original. 